\title{
Mixed Finite Element Methods of Higher-Order for Model Contact Problems
}

\author{
Andreas Schröder
}

\begin{abstract}
This paper presents mixed finite element methods of higher-order for a simplified Signorini problem and an idealized frictional problem. The discretization is based on a mixed variational formulation proposed by Haslinger et al. which is extended to higher-order finite elements. To guarantee the unique existence of the solution of the mixed method, a discrete inf-sup condition is proven. Approximation results of the $p$-method of finite elements and some inverse estimates for higher-order polynomials are applied. Numerical results confirm the theoretical findings.
\end{abstract}

Keywords higher-order FEM · contact problems $\cdot$ mixed methods

\section{Introduction}

The aim of this paper is to derive mixed finite element methods of higher-order for contact problems which play an important role in mechanical engineering, $[6,7,11]$. Here, we consider a simplified Signorini problem and an idealized frictional problem as model problems. The discretization approach is based on mixed finite elements for contact problems introduced by Haslinger et al. in [8-10]. This approach is originally developed for lower-order finite elements. In this paper, we extend it to higher-order finite elements. The approach relies on a saddle point formulation where the geometrical contact condition and the frictional conditions are captured by Lagrange multipliers. The restrictions for the Lagrange multipliers are sign conditions or box constraints and are, therefore, more simple than the original contact conditions. However, the Lagrange multipliers are additional variables which also have to be discretized. Whereas the unique existence of a saddle point is ensured

\author{
A. Schröder \\ Unter den Linden 6 \\ 10099 Berlin, Germany \\ Tel.: +49-2093-2630 \\ Fax: +49-2093-5859 \\ E-mail: andreas.schroeder@mathematik.hu-berlin.de
}


for the non-discretized problem, we can not generally ensure this for the discretized Lagrange multlipliers. In many mixed formulations, unique existence follows from a inf-sup condition associated to the discretization spaces. But its verification is often a crucial point. For lower-order finite elements, the inf-sup condition for the introduced contact problems is proven in the above mentioned references. In this work, we prove the inf-sup condition for higher-order finite elements using approximation results for the $p$-method of finite elements, and recently published inverse estimates for higher order polynomials, $[1,5]$.

An important assumption of the proof is that the used approach allows for the discretization of the Lagrange multipliers on boundary meshes with a larger mesh size than that of the primal variable. In pratice, this leads to a high implementational effort. We refer to [2] for a mixed finite element scheme which avoids different meshes. In general, higher-order discretization schemes for contact problems are rarely studied in literature, especially for mixed variational formulation. For discretization techniques based on a primal, non-mixed formulations, we refer to [12,13].

This paper is organized as follows: In Sections 3 and 4, the mixed variational formulations are introduced for the simplified Signorini problem and idealized frictional problem. Higher-order finite element discretizations based on the mixed formulations are presented in Section 5. The main part of this work, the derivation of the inf-sup condition for higher-order finite elements, is proposed in Section 6. Numerical results confirming the theoretical findings are presented in Section 7.

\section{Notation}

Let $\Omega \subset \mathbb{R}^{k}, k \in \mathbb{N}$, be a domain with sufficiently smooth boundary $\Gamma:=\partial \Omega$. Moreover, let $\Gamma_{D} \subset \Gamma$ be closed with positive measure and let $\Gamma_{C} \subset \Gamma \backslash \Gamma_{D}$ with $\bar{\Gamma}_{C} \varsubsetneqq \Gamma \backslash \Gamma_{D}$ and $\Gamma_{N}:=\Gamma \backslash\left(\Gamma_{D} \cup \bar{\Gamma}_{C}\right) . L^{2}(\Omega), H^{l}(\Omega), l \geq 1$, and $H^{1 / 2}\left(\Gamma_{C}\right)$ denote the usual Sobolev spaces and

$$
H^{1}\left(\Omega, \Gamma_{D}\right):=\left\{v \in H^{1}(\Omega) \mid \gamma(v)=0 \text { on } \Gamma_{D}\right\}
$$

with the trace operator $\gamma$. The space $H^{-1 / 2}\left(\Gamma_{C}\right)$ denotes the topological dual space of $H^{1 / 2}\left(\Gamma_{C}\right)$ with the norms $\|\cdot\|_{-1 / 2, \Gamma_{C}}$ and $\|\cdot\|_{1 / 2, \Gamma_{C}}$, respectively. Let $(\cdot, \cdot)_{0, \omega}$, $(\cdot, \cdot)_{0, \Gamma^{\prime}}$ be the usual $L^{2}$-scalar products on $\omega \subset \Omega$ and $\Gamma^{\prime} \subset \Gamma$, respectively. We define $\|v\|_{0, \omega}^{2}:=(v, v)_{0, \omega}$ and omit the subscript $\omega$ whenever $\omega=\Omega$. Moreover, we state

$$
|v|_{1}^{2}:=(\nabla v, \nabla v)_{0}, \quad\|v\|_{1}^{2}:=\|v\|_{0}^{2}+|v|_{1}^{2}
$$

as the usual, equivalent $H^{1}$-norms on $H^{1}\left(\Omega, \Gamma_{D}\right)$ with the gradient operator $\nabla$ in the weak sense. We denote the usual Laplace operator likewise in the weak sense by $\Delta$. Note, the linear and bounded mapping

$$
\gamma_{C}:=\gamma_{\Gamma_{C}}: H^{1}\left(\Omega, \Gamma_{D}\right) \rightarrow H^{1 / 2}\left(\Gamma_{C}\right)
$$

is surjective due to the assumptions on $\Gamma_{C}$, [11]. As these assumptions are fulfilled in most cases, we can avoid the introduction of complicated $H_{00}^{1 / 2}\left(\Gamma_{C}\right)$-spaces. For functions in $L^{2}(\Omega)$ or $L^{2}\left(\Gamma_{C}\right)$, the inequality symbols $\geq$ and $\leq$ are defined by means of "almost everywhere". 


\section{Mixed variational formulation of a simplified Signorini problem}

A simplified Signorini problem is to find a function $u \in H^{1}\left(\Omega, \Gamma_{D}\right) \cap H^{2}(\Omega)$ such that

$$
\begin{aligned}
&-\Delta u=f \quad \text { in } \Omega, \\
& \partial_{n} u=0 \quad \text { on } \Gamma_{N}, \\
& u \geq g, \quad \partial_{n} u \geq 0, \quad \partial_{n} u(u-g)=0 \quad \text { on } \Gamma_{C},
\end{aligned}
$$

where $f \in L^{2}(\Omega)$. The function $g \in H^{1 / 2}\left(\Gamma_{C}\right)$ represents an obstacle on the boundary $\Gamma_{C}$. It is well-known, that $u \in H^{1}\left(\Omega, \Gamma_{D}\right) \cap H^{2}(\Omega)$ is a solution of the simplified Signorini problem if and only if $u \in K:=\left\{v \in H^{1}\left(\Omega, \Gamma_{D}\right) \mid \gamma(v) \geq g\right.$ on $\left.\Gamma_{C}\right\}$ and

$$
\forall v \in K:(\nabla u, \nabla(v-u))_{0} \geq(f,(v-u))_{0} .
$$

Moreover, $u \in H^{1}\left(\Omega, \Gamma_{D}\right)$ fulfills (2) if and only if $u$ is a minimizer of the functional

$$
E(v):=\frac{1}{2}(\nabla v, \nabla v)_{0}-(f, v)_{0}
$$

in $K$. The functional $E$ is strictly convex, continuous and coercive due to Cauchy's and Poincare's inequalities. This implies the unique existence of a minimizer $u$.

In order to derive a mixed formulation, let

$$
\begin{aligned}
H_{-}^{1 / 2}\left(\Gamma_{C}\right) & :=\left\{w \in H^{1 / 2}\left(\Gamma_{C}\right) \mid w \leq 0\right\}, \\
H_{-}^{-1 / 2}\left(\Gamma_{C}\right) & :=\left\{\mu \in H^{-1 / 2}\left(\Gamma_{C}\right) \mid \forall w \in H_{-}^{1 / 2}\left(\Gamma_{C}\right):\langle\mu, w\rangle \geq 0\right\} .
\end{aligned}
$$

Using the Hahn-Banach theorem it can be proven that

$$
\sup _{\mu_{0} \in H_{-}^{-1 / 2}\left(\Gamma_{C}\right)}\left\langle\mu_{0}, \gamma_{C}(v)-g\right\rangle= \begin{cases}0, & \text { if } v \in K \\ \infty, & \text { else. }\end{cases}
$$

Therefore, we obtain

$$
E(u)=\inf _{v \in H^{1}\left(\Omega, \Gamma_{D}\right)} \sup _{\mu_{0} \in H_{-}^{-1 / 2}\left(\Gamma_{C}\right)} \mathscr{L}_{0}\left(v, \mu_{0}\right)
$$

with the Lagrange functional

$$
\mathscr{L}_{0}\left(v, \mu_{0}\right):=E(v)+\left\langle\mu_{0}, \gamma_{C}(v)-g\right\rangle
$$

on $H^{1}\left(\Omega, \Gamma_{D}\right) \times H_{-}^{-1 / 2}\left(\Gamma_{C}\right)$. This states, whenever $\left(u, \lambda_{0}\right) \in H^{1}\left(\Omega, \Gamma_{D}\right) \times H_{-}^{1 / 2}\left(\Gamma_{C}\right)$ is a saddle point of $\mathscr{L}_{0}$, then $u$ is a minimizer of $E$.

The existence of a unique saddle point is guaranteed, if there exists a constant $\alpha>0$ such that

$$
\alpha\|\mu\|_{-1 / 2, \Gamma_{C}} \leq \sup _{\substack{v \in H^{1}\left(\Omega, \Gamma_{D}\right) \\\|v\|_{1}=1}}\left\langle\mu, \gamma_{C}(v)\right\rangle
$$

is fulfilled for all $\mu \in H^{-1 / 2}\left(\Gamma_{C}\right)$, [11]. In fact, it follows from the closed range theorem and the surjectivity of $\gamma_{C}$, that (3) is valid. 
Let $\mathscr{L}_{0, \lambda_{0}}^{\prime}: H^{1}\left(\Omega, \Gamma_{D}\right) \rightarrow\left(H^{1}\left(\Omega, \Gamma_{D}\right)\right)^{*}$ and $\mathscr{L}_{0, u}^{\prime}: H^{-1 / 2}\left(\Gamma_{C}\right) \rightarrow\left(H^{-1 / 2}\left(\Gamma_{C}\right)\right)^{*} \simeq$ $H^{1 / 2}\left(\Gamma_{C}\right)$ be the Fréchet derivatives of $\mathscr{L}_{0, \lambda_{0}}:=\mathscr{L}_{0}\left(\cdot, \lambda_{0}\right)$ and $\mathscr{L}_{0, u}:=\mathscr{L}_{0}(u, \cdot)$, respectively. Then, $\left(u, \lambda_{0}\right) \in H^{1}\left(\Omega, \Gamma_{D}\right) \times H_{-}^{-1 / 2}\left(\Gamma_{C}\right)$ is a saddle point of $\mathscr{L}_{0}$, if and only if the stationary conditions $\mathscr{L}_{0, \lambda_{0}}^{\prime}(u)=0$ and $\left\langle\mu_{0}-\lambda_{0}, \mathscr{L}_{u}^{\prime}\left(\lambda_{0}\right)\right\rangle \leq 0$ for $\mu_{0} \in$ $H_{-}^{-1 / 2}\left(\Gamma_{C}\right)$ are fulfilled. Thus, $\left(u, \lambda_{0}\right)$ is equivalently characterized by the mixed variational formulation

$$
\begin{aligned}
& \forall v \in H^{1}\left(\Omega, \Gamma_{D}\right):(\nabla u, \nabla v)_{0}=(f, v)_{0}-\left\langle\lambda_{0}, \gamma_{C}(v)\right\rangle, \\
& \forall \mu_{0} \in H_{-}^{-1 / 2}\left(\Gamma_{C}\right):\left\langle\mu_{0}-\lambda_{0}, \gamma_{C}(u)-g\right\rangle \leq 0 .
\end{aligned}
$$

\section{Mixed variational formulation of an idealized frictional problem}

An idealized frictional problem is to find a function $u \in H^{1}\left(\Omega, \Gamma_{D}\right) \cap H^{2}(\Omega)$ such that

$$
\begin{aligned}
-\Delta u=f & \text { in } \Omega, \\
\partial_{n} u=0 & \text { on } \Gamma_{N}, \\
\left|\partial_{n} u\right| \leq s & \text { with }\left\{\begin{array}{l}
\left|\partial_{n} u\right|<s \Rightarrow u=0, \\
\partial_{n} u=s \Rightarrow u \geq 0, \\
-\partial_{n} u=s \Rightarrow u \leq 0
\end{array}\right\} \text { on } \Gamma_{C}
\end{aligned}
$$

for $f \in L^{2}(\Omega)$ and $s \in L^{2}\left(\Gamma_{C}\right), s \geq 0$. Here, $u \in H^{1}\left(\Omega, \Gamma_{D}\right) \cap H^{2}(\Omega)$ is a solution if and only if

$$
\forall v \in H^{1}\left(\Omega, \Gamma_{D}\right):(\nabla u, \nabla(v-u))_{0}+(s,|\gamma(v)|-|\gamma(u)|)_{0, \Gamma_{C}} \geq(f, v-u)_{0} .
$$

It is well-known, that $u \in H^{1}\left(\Omega, \Gamma_{D}\right)$ fulfills (5) if and only if $u$ is a minimizer of the (non-differentiable) functional $E+j$ in $H^{1}\left(\Omega, \Gamma_{D}\right)$ with $j(v):=\left(s,\left|\gamma_{C}(v)\right|\right)_{0, \Gamma_{C}}$, [7]. Since $j$ is strictly convex, continuous and coercive, the unique existence of a minimizer $u$ is guaranteed.

We define

$$
L_{1}^{2}\left(\Gamma_{C}\right):=\left\{\mu_{1} \in L^{2}\left(\Gamma_{C}\right)|| \mu_{1} \mid \leq 1 \text { on supp } s, \mu_{1}=0 \text { on } \Gamma_{C} \backslash \operatorname{supp} s\right\} .
$$

For $\mu_{1} \in L_{1}^{2}\left(\Gamma_{C}\right)$ and $v \in H^{1}\left(\Omega, \Gamma_{D}\right)$, there holds $\left(\mu_{1}, s \gamma_{C}(v)\right)_{0, \Gamma_{C}} \leq\left(\left|\mu_{1}\right|, s\left|\gamma_{C}(v)\right|\right)_{0, \Gamma_{C}} \leq$ $j(v)$. Furthermore, we have

$$
j(v)=\int_{\Gamma_{C}} \tilde{\mu}_{1} s \gamma_{C}(v) d \Gamma \leq \sup _{\mu_{1} \in L_{1}^{2}\left(\Gamma_{C}\right)}\left(\mu_{1}, s \gamma_{C}(v)\right)_{0, \Gamma_{C}}
$$

with $\tilde{\mu}_{1}:=\operatorname{sign}\left(\gamma_{C}(v)\right)$ on supp $s$ and $\tilde{\mu}_{1}:=0$ on $\Gamma_{C} \backslash \operatorname{supp} s$. In conclusion, we obtain

$$
j(v)=\sup _{\mu_{1} \in L_{1}^{2}\left(\Gamma_{C}\right)}\left(\mu_{1}, s \gamma_{C}(v)\right)_{0, \Gamma_{C}}
$$


and

$$
(E+j)(u)=\inf _{v \in H^{1}\left(\Omega, \Gamma_{D}\right)} \sup _{\mu_{1} \in L_{1}^{2}\left(\Gamma_{C}\right)} \mathscr{L}_{1}\left(v, \mu_{1}\right)
$$

with the Lagrange functional

$$
\mathscr{L}_{1}\left(v, \mu_{1}\right):=E(v)+\left(\mu_{1}, s \gamma_{C}(v)\right)_{0, \Gamma_{C}}
$$

on $H^{1}\left(\Omega, \Gamma_{D}\right) \times L_{1}^{2}\left(\Gamma_{C}\right)$. Thus, whenever $\left(u, \lambda_{1}\right) \in H^{1}\left(\Omega, \Gamma_{D}\right) \times L_{1}^{2}\left(\Gamma_{C}\right)$ is a saddle point of $\mathscr{L}_{1}$, then $u$ is a minimizer of $E+j$. Due to the boundness of $L_{1}^{2}\left(\Gamma_{C}\right)$, the existence of a minimizer is guaranteed, [4].

In analogy to the simplified Signorini problem, the pair $\left(u, \lambda_{1}\right) \in H^{1}\left(\Omega, \Gamma_{D}\right) \times L_{1}^{2}\left(\Gamma_{C}\right)$ is equivalently characterized by the mixed variational formulation,

$$
\begin{gathered}
\forall v \in H^{1}\left(\Omega, \Gamma_{D}\right):(\nabla u, \nabla v)_{0}=(f, v)_{0}-\left(\lambda_{1}, s \gamma_{C}(v)\right)_{0, \Gamma_{C}} \\
\forall \mu \in L_{1}^{2}\left(\Gamma_{C}\right):\left(\mu_{1}-\lambda_{1}, s \gamma_{C}(u)\right)_{0, \Gamma_{C}} \leq 0 .
\end{gathered}
$$

Since $H^{1 / 2}\left(\Gamma_{C}\right)$ is dense in $L^{2}\left(\Gamma_{C}\right)$, we conclude from (6) that the Lagrange multiplier is unique, too.

An alternative mixed formulation for the idealized frictional problem is given through the definition of

$$
L_{s}^{2}\left(\Gamma_{C}\right):=\left\{\mu_{1} \in L^{2}\left(\Gamma_{C}\right)|| \mu_{1} \mid \leq s\right\}
$$

We obtain

$$
j(v)=\sup _{\mu_{1} \in L_{S}^{2}\left(\Gamma_{C}\right)}\left(\mu_{1}, \gamma_{C}(v)\right)_{0, \Gamma_{C}}
$$

by similar arguments as above. Therefore, we have

$$
(E+j)(u)=\inf _{v \in H^{1}\left(\Omega, \Gamma_{D}\right)} \sup _{\mu_{1} \in L_{S}^{2}\left(\Gamma_{C}\right)} \tilde{\mathscr{L}}_{1}\left(v, \mu_{1}\right)
$$

with the Lagrange functional

$$
\tilde{\mathscr{L}}_{1}\left(v, \mu_{1}\right):=E(v)+\left(\mu_{1}, \gamma_{C}(v)\right)_{0, \Gamma_{C}}
$$

on $H^{1}\left(\Omega, \Gamma_{D}\right) \times L_{s}^{2}\left(\Gamma_{C}\right)$. In this case, the pair $\left(u, \lambda_{1}\right) \in H^{1}\left(\Omega, \Gamma_{D}\right) \times L_{s}^{2}\left(\Gamma_{C}\right)$ is equivalently characterized by

$$
\begin{aligned}
& \forall v \in H^{1}\left(\Omega, \Gamma_{D}\right):(\nabla u, \nabla v)_{0}=(f, v)_{0}-\left(\lambda_{1}, \gamma_{C}(v)\right)_{0, \Gamma_{C}}, \\
& \forall \mu \in L_{s}^{2}\left(\Gamma_{C}\right):\left(\mu_{1}-\lambda_{1}, \gamma_{C}(u)\right)_{0, \Gamma_{C}} \leq 0 .
\end{aligned}
$$




\section{Higher-order discretization of the mixed variational formulations}

We propose a higher-order finite element discretization based on quadrangles or hexahedrons as follows: Let $\mathscr{T}_{h}$ and $\mathscr{T}_{C, H}$ be finite element meshes of $\Omega$ and $\Gamma_{C}$ with mesh sizes $h$ and $H$, respectively. Let $\Psi_{T}:[-1,1]^{k} \rightarrow T \in \mathscr{T}_{h}, \Psi_{C, T_{C}}:[-1,1]^{k-1} \rightarrow$ $T_{C} \in \mathscr{T}_{C, H}$ be bijective and sufficiently smooth transformations and let $p_{T}, p_{C, T_{C}} \in \mathbb{N}$ be degree distributions on $\mathscr{T}_{h}$ and $\mathscr{T}_{C, H}$, respectively. Using the polynomial tensor product space $S_{k}^{q}$ of order $q$ on the reference element $[-1,1]^{k}$, we define

$$
S^{p}\left(\mathscr{T}_{h}\right):=\left\{v \in H^{1}\left(\Omega, \Gamma_{D}\right) \mid \forall T \in \mathscr{T}_{h}: v_{\mid T} \circ \Psi_{T} \in S_{k}^{p_{T}}\right\}
$$

and

$$
M^{p_{C}}\left(\mathscr{T}_{C, H}\right):=\left\{\mu \in L^{2}\left(\Gamma_{C}\right) \mid \forall T_{C} \in \mathscr{T}_{C, H}: \mu_{\mid T_{C}} \circ \Psi_{C, T_{C}} \in S_{k-1}^{p_{C, T_{C}}}\right\} .
$$

Moreover, we define

$M_{-}^{p_{C}}\left(\mathscr{T}_{C, H}\right):=\left\{\mu_{0, H} \in M^{p_{C}}\left(\mathscr{T}_{C, H}\right) \mid \mu_{0, H} \leq 0\right\}$,

$M_{1}^{p_{C}}\left(\mathscr{T}_{C, H}\right):=\left\{\mu_{1, H} \in M^{p_{C}}\left(\mathscr{T}_{C, H}\right)|| \mu_{1, H} \mid \leq 1\right.$ on $\operatorname{supp} s, \mu_{1, H}=0$ on $\left.\Gamma_{C} \backslash \operatorname{supp} s\right\}$, $M_{s}^{p_{C}}\left(\mathscr{T}_{C, H}\right):=\left\{\mu_{1, H} \in M^{p_{C}}\left(\mathscr{T}_{C, H}\right)|| \mu_{1, H} \mid \leq s\right\}$.

The discrete saddle problem of the simplified Signorini problem consists in finding a discrete saddle point $\left(u_{h}, \lambda_{0, H}\right) \in S^{p}\left(\mathscr{T}_{h}\right) \times M_{-}^{p_{C}}\left(\mathscr{T}_{C, H}\right)$, such that

$$
\mathscr{L}_{0}\left(u_{h}, \lambda_{0, H}\right)=\inf _{v_{h} \in S^{p}\left(\mathscr{T}_{h}\right)} \sup _{\mu_{0, H} \in M_{-}^{p_{C}}\left(\mathscr{T}_{C, H}\right)} \mathscr{L}_{0}\left(v_{h}, \mu_{0, H}\right)
$$

It is easy to see, that the first component of the discrete saddle point is the unique minimizer of the minimization problem

$$
E\left(u_{h}\right)=\min _{v_{h} \in K_{h H}} E\left(v_{h}\right)
$$

with $K_{h H}:=\left\{v_{h} \in S^{p}\left(\mathscr{T}_{h}\right) \mid \forall \mu_{0, H} \in M_{-}^{p_{C}}\left(\mathscr{T}_{C, H}\right):\left(\mu_{0, H}, \gamma_{C}\left(v_{h}\right)-g\right)_{0, \Gamma_{C}} \leq 0\right\}$. By the stationary condition, we conclude that the discrete saddle point is equivalently characterized by

$$
\begin{aligned}
& \forall v_{h} \in S^{p}\left(\mathscr{T}_{h}\right):\left(\nabla u_{h}, \nabla v_{h}\right)_{0}=\left(f, v_{h}\right)_{0}-\left(\lambda_{0, H}, \gamma_{C}\left(v_{h}\right)\right)_{0, \Gamma_{C}}, \\
& \forall \mu_{0, H} \in M_{-}^{p_{C}}\left(\mathscr{T}_{C, H}\right):\left(\mu_{0, H}-\lambda_{0, H}, \gamma_{C}\left(u_{h}\right)-g\right)_{0, \Gamma_{C}} \leq 0 .
\end{aligned}
$$

Following the approach of Oden et al., [11, Remark 3.4.3] and [15], we conclude

Theorem 1 Let $g \in \gamma_{C}\left(S^{p}\left(\mathscr{T}_{h}\right)\right)$, then there exists a discrete saddle point of the simplified Signorini problem.

Proof. Due to the closeness of $\gamma_{C}\left(S^{p}\left(\mathscr{T}_{h}\right)\right)$ in $H^{1 / 2}\left(\Gamma_{C}\right)$, we obtain from the closed range theorem, [17], that there exists a constant $\alpha>0$ such that

$$
\alpha\|[\mu]\| \leq \sup _{v_{h} \in V_{h},\left\|v_{h}\right\|=1}\left\langle\mu, \gamma_{C}\left(v_{h}\right)\right\rangle
$$


for all $[\mu] \in H^{-1 / 2}\left(\Gamma_{C}\right) / \operatorname{ker} \gamma_{C \mid S^{p}\left(\mathscr{T}_{h}\right)}^{\prime}$ where $[\mu]:=\mu+\operatorname{ker} \gamma_{C \mid S^{p}\left(\mathscr{T}_{h}\right)}$, and $\|[\mu]\|:=$ $\inf _{\kappa \in \operatorname{ker} \gamma_{C \mid S p}^{\prime}\left(\mathscr{T}_{h}\right)}\|\mu+\kappa\|_{1 / 2, \Gamma_{C}}$ and $\gamma_{C \mid S^{p}\left(\mathscr{T}_{h}\right)}^{\prime}: H^{-1 / 2}\left(\Gamma_{C}\right) \rightarrow S^{p}\left(\mathscr{T}_{h}\right)^{\prime}$ denotes the transpose of $\gamma_{C \mid S^{p}\left(\mathscr{T}_{h}\right)}$. We define

$$
\hat{\mathscr{L}}\left(v_{h},[\mu]\right):=E\left(v_{h}\right)+\left\langle\mu, \gamma_{C}\left(v_{h}\right)-g\right\rangle,
$$

which is well-defined due to $g \in \gamma_{C}\left(S^{p}\left(\mathscr{T}_{h}\right)\right)$. Using (10), we conclude by standard arguments (e.g. [11, Lem. 3.2]), that

$$
H^{-1 / 2}\left(\Gamma_{C}\right) / \operatorname{ker} \gamma_{C \mid S^{p}\left(\mathscr{T}_{h}\right)}^{\prime} \ni[\mu] \mapsto \sup _{v_{h} \in S^{p}\left(\mathscr{T}_{h}\right)}-\hat{\mathscr{L}}\left(v_{h},[\mu]\right)
$$

is coercive. We set

$$
\hat{M}_{-}^{p_{C}}\left(\mathscr{T}_{C, H}\right):=\left\{\left[\mu_{0, H}\right] \in H^{-1 / 2}\left(\Gamma_{C}\right) / \operatorname{ker} \gamma_{C \mid S^{p}\left(\mathscr{T}_{h}\right)}^{\prime} \mid \mu_{0, H} \in M_{-}^{p_{C}}\left(\mathscr{T}_{C, H}\right)\right\}
$$

which is a closed and convex set. Due to [4, Prop IV.2.3 and Remark IV.2.1], there exists $\left(u_{h},\left[\lambda_{0, H}\right]\right) \in S^{p}\left(\mathscr{T}_{h}\right) \times \hat{M}_{-}^{p_{C}}\left(\mathscr{T}_{C, H}\right)$ with

$$
\hat{\mathscr{L}}\left(u_{h},\left[\lambda_{0, H}\right]\right)=\inf _{v_{h} \in S^{p}\left(\mathscr{T}_{h}\right)} \sup _{\mu_{0, H} \in \hat{M}_{-}^{p}\left(\mathscr{T}_{C, H}\right)} \hat{\mathscr{L}}\left(v_{h},\left[\mu_{0, H}\right]\right) .
$$

Thus, $\left(u_{h}, \lambda_{0, H}\right)$ fulfills ( 8$)$.

Remark 1 Theorem 1 is an alternative to the existence result established by Hlavacek et al. [10, Lem. 5.6], where $K_{h H}^{\circ} \neq \emptyset$ instead of $g \in \gamma_{C}\left(S^{p}\left(\mathscr{T}_{h}\right)\right)$.

Theorem 1 does not imply the uniqueness of a saddle point. Furthermore, the existence of a saddle point depends on the assumption $g \in \gamma_{C}\left(S^{p}\left(\mathscr{T}_{h}\right)\right)$ which is not fulfilled in general. Condition (10) is based on the closeness of $\gamma_{C}\left(S^{p}\left(\mathscr{T}_{h}\right)\right)$ and requires to consider a saddle point problem in $S^{p}\left(\mathscr{T}_{h}\right) \times H^{-1 / 2}\left(\Gamma_{C}\right) / \operatorname{ker} \gamma_{C \mid S^{p}\left(\mathscr{T}_{h}\right)}^{\prime}$. Hence, it is more natural to directly claim the inf-sup condition for $S^{p}\left(\mathscr{T}_{h}\right) \times M^{p_{C}}\left(\mathscr{T}_{C, H}\right)$.

Theorem 2 If there is a constant $\alpha>0$ such that

$$
\alpha\left\|\mu_{H}\right\|_{-1 / 2, \Gamma_{C}} \leq \sup _{\substack{v_{h} \in S^{P}\left(\mathscr{S}_{h}\right) \\\left\|v_{h}\right\|_{1}=1}}\left(\mu_{H}, \gamma_{C}\left(v_{h}\right)\right)_{0, \Gamma_{C}}
$$

for all $\mu_{H} \in M^{p_{C}}\left(\mathscr{T}_{C, H}\right)$, then there exists a unique discrete saddle point of the simplified Signorini problem.

Proof. Analogously to the proof of Theorem 1, we conclude that

$$
M^{p_{C}}\left(\mathscr{T}_{C, H}\right) \ni \mu_{H} \mapsto \sup _{v_{h} \in S^{p}\left(\mathscr{T}_{h}\right)}-\mathscr{L}_{0}\left(v_{h}, \mu_{H}\right)
$$

is coercive. This implies the existence of a saddle point. The uniqueness is a direct consequence of (11). 
The discrete saddle point problem of the idealized frictional problem is to find a pair $\left(u_{h}, \lambda_{1, H}\right) \in S^{p}\left(\mathscr{T}_{h}\right) \times M_{1}^{p_{C}}\left(\mathscr{T}_{C, H}\right)$ such that

$$
\mathscr{L}_{1}\left(u_{h}, \lambda_{1, H}\right)=\inf _{v_{h} \in S^{p}\left(\mathscr{T}_{h}\right)} \sup _{\mu_{1, H} \in M_{1}^{p_{C}}\left(\mathscr{T}_{C, H}\right)} \mathscr{L}_{1}\left(v_{h}, \mu_{1, H}\right)
$$

The first component is the unique minimizer of the minimization problem

$$
E\left(u_{h}\right)=\min _{v_{h} \in S^{p}\left(\mathscr{T}_{h}\right)} E\left(v_{h}\right)+j_{h H}\left(v_{h}\right),
$$

where $j_{h H}\left(v_{h}\right):=\sup _{\mu_{1, H} \in M_{1}^{p} C}\left(\mathscr{T}_{C, H}\right)\left(\mu_{1, H}, s \gamma_{C}\left(v_{h}\right)\right)_{0, \Gamma_{C}}$. The discrete saddle point is equivalently characterized by

$$
\begin{aligned}
& \forall v_{h} \in S^{p}\left(\mathscr{T}_{h}\right):\left(\nabla u_{h}, \nabla v_{h}\right)_{0}=\left(f, v_{h}\right)_{0}-\left(\lambda_{1, H}, s \gamma_{C}\left(v_{h}\right)\right)_{0, \Gamma_{C}}, \\
& \forall \mu_{1, H} \in M_{1}^{p_{C}}\left(\mathscr{T}_{C, H}\right):\left(\mu_{1, H}-\lambda_{1, H}, s \gamma_{C}\left(u_{h}\right)\right)_{0, \Gamma_{C}} \leq 0 .
\end{aligned}
$$

Alternatively, we may seek a discrete saddle point $\left(u_{h}, \lambda_{1, H}\right) \in S^{p}\left(\mathscr{T}_{h}\right) \times M_{S}^{p_{C}}\left(\mathscr{T}_{C, H}\right)$, such that

$$
\tilde{\mathscr{L}}_{1}\left(u_{h}, \lambda_{1, H}\right)=\inf _{v_{h} \in S^{p}\left(\mathscr{T}_{h}\right)} \sup _{\mu_{1, H} \in M_{s}^{p}\left(\mathscr{T}_{C, H}\right)} \tilde{\mathscr{L}}_{1}\left(v_{h}, \mu_{1, H}\right) .
$$

Again, the first component $u_{h}$ is the unique minimizer of

$$
\left(E+\tilde{j}_{h H}\right)\left(u_{h}\right)=\min _{v_{h} \in S^{p}\left(\mathscr{T}_{h}\right)} E\left(v_{h}\right)+\tilde{j}_{h H}\left(v_{h}\right) .
$$

where $\tilde{j}_{h H}:=\sup _{\mu_{1, H} \in M_{s}^{p} C\left(\mathscr{T}_{C, H}\right)}\left(\mu_{1, H}, \gamma_{C}\left(v_{h}\right)\right)_{0, \Gamma_{C}}$. Hence, the discrete saddle point is equivalently characterized by

$$
\begin{aligned}
& \forall v_{h} \in S^{p}\left(\mathscr{T}_{h}\right):\left(\nabla u_{h}, \nabla v_{h}\right)_{0}=\left(f, v_{h}\right)_{0}-\left(\lambda_{1, H}, \gamma_{C}\left(v_{h}\right)\right)_{0, \Gamma_{C}}, \\
& \forall \mu_{1, H} \in M_{s}^{p_{C}}\left(\mathscr{T}_{C, H}\right):\left(\mu_{1, H}-\lambda_{1, H}, \gamma_{C}\left(u_{h}\right)\right)_{0, \Gamma_{C}} \leq 0 .
\end{aligned}
$$

Theorem 3 There exist discrete saddle points of (12) and (14). The discrete saddle point of (14) is unique if (11) is fulfilled.

Proof. The sets $M_{1}^{p_{C}}\left(\mathscr{T}_{C, H}\right)$ and $M_{S}^{P_{C}}\left(\mathscr{T}_{C, H}\right)$ are bounded. The existence of a discrete saddle point of (12) and (14) is guaranteed by [4, Prop IV.2.3 and Remark IV.2.1]. Let $\left(u, \lambda_{1, H}\right),\left(u, \lambda_{1, H}^{*}\right) \in S^{p}\left(\mathscr{T}_{h}\right) \times M_{1}^{p_{C}}\left(\mathscr{T}_{C, H}\right)$ be discrete saddle points of (12). From (11) we obtain $\left\|\lambda_{1, H}-\lambda_{1, H}^{*}\right\|_{-1 / 2, \Gamma_{C}}=0$. Since $H^{-1 / 2}\left(\Gamma_{C}\right)$ is dense in $L^{2}\left(\Gamma_{C}\right)$, we have $\lambda_{1, H}=\lambda_{1, H}^{*}$.

Remark 2 The uniqueness of the discrete saddle point of (12) is not a direct consequence of (11). We refer to the end of Section 6 for a proof of uniqueness under further assumptions.

We call the discretization schemes (9), (13) and (15) stable, if there exists a unique discrete saddle point independently of the discretization level. In other words, to guarantee the discretization schemes (9) and (15) to be stable, the constant in (11) has to be independent of $h, H, p$ and $p_{C}$. In [10], the discrete inf-sup condition (11) is proven with an $h$ - and $H$-independent constant $\alpha$ for uniform meshes and $p \equiv 1, p_{C} \equiv 0$. The essential assumption is that the quotient $h / H$ is sufficiently small. In the next section, we will show, that these results can be carried over to the proposed higher-order schemes. 


\section{The inf-sup condition for higher-order discretizations}

In this section, we show condition (11) for discretization schemes of higher-order. In particular, we show that the constant $\alpha$ can be chosen independently from $h, H, p$ and $p_{C}$. Therefor, we make use of an approximation result for higher-order finite element methods (Lemma 2) and for an inverse inequality for negative norms (Lemma 3) which was recently pubished by Georgoulis, [5]. Furthermore, we follow the proof of Lemma 3.1 in [9] where this condition is derived for discretization schemes of lower-order.

The interpolation spaces $H^{1+\theta}(\Omega)$ and $H^{-1 / 2+\theta}\left(\Gamma_{C}\right)$ are defined via

$$
H^{1+\theta}(\Omega):=\left[H^{1}(\Omega), H^{2}(\Omega)\right]_{\theta, 2}
$$

and

$$
\left.H^{-1 / 2+\theta}\left(\Gamma_{C}\right):=\left[H^{-1 / 2}\left(\Gamma_{C}\right), H^{1 / 2}\left(\Gamma_{C}\right)\right)\right]_{\theta, 2}
$$

with norms $\|\cdot\|_{1+\theta}$ and $\|\cdot\|_{-1 / 2+\theta, \Gamma_{C}}$, respectively, where $0<\theta \leq 1,[14,16]$.

In this work, we focus on the two-dimensional case $(k=2)$ and assume that $\mathscr{T}_{h}$ is a quasi-uniform parallelogram mesh. For the mesh $\mathscr{T}_{C, H}$ consisting of line segments, we assume

$$
\forall T_{C} \in \mathscr{T}_{C, H}: \kappa H \leq H_{T_{C}}
$$

with a constant $\kappa>0$ which is idenpendent of $H$. Here, $H_{T_{C}}$ denotes the length of the line segment $T_{C}$. Moreover, we assume that $p$ and $p_{C}$ are constant degree distributions.

Lemma 1 For $\mu \in H^{-1 / 2}\left(\Gamma_{C}\right)$, there exists a function $u^{\mu} \in H^{1}\left(\Omega, \Gamma_{D}\right)$ such that

$$
\left(\nabla u^{\mu}, \nabla v\right)_{0}+\left(u^{\mu}, v\right)_{0}=\left\langle\mu, \gamma_{C}(v)\right\rangle
$$

for all $v \in H^{1}\left(\Omega, \Gamma_{D}\right)$. Additionally, there holds $C_{1}\|\mu\|_{-1 / 2, \Gamma_{C}} \leq\left\|u^{\mu}\right\|_{1}$ for a constant $C_{1}>0$.

Proof. The existence of $u^{\mu} \in H^{1}\left(\Omega, \Gamma_{D}\right)$ is guaranteed by the Lax-Milgram Lemma. The mapping $\hat{\gamma}_{C}: H^{1}\left(\Omega, \Gamma_{D}\right) / \operatorname{ker} \gamma_{C} \rightarrow H^{1 / 2}\left(\Gamma_{C}\right)$ with $\hat{\gamma}_{C}([v]):=\gamma_{C}(v)$ and $[v]:=$ $v+\operatorname{ker} \gamma_{C}$ is bijective and continuous. Since $H^{1}\left(\Omega, \Gamma_{D}\right)$ and $H^{1 / 2}\left(\Gamma_{C}\right)$ are Banach spaces, the inverse $\hat{\gamma}_{C}^{-1}$ is continuous, too. Let

$$
\left\|\hat{\gamma}_{C}^{-1}\right\|:=\sup _{\substack{w \in H^{1 / 2}\left(\Gamma_{C}\right) \\\|w\|_{1 / 2, \Gamma_{C}}=1}}\left\|\hat{\gamma}_{C}^{-1}(w)\right\|
$$

with $\|[v]\|:=\inf _{w \in \operatorname{ker} \gamma_{C}}\|v+w\|_{1}$ for $[v] \in H^{1}\left(\Omega, \Gamma_{D}\right) / \operatorname{ker} \gamma_{C}$ and let

$$
V:=\left\{v \in H^{1}\left(\Omega, \Gamma_{C}\right) \mid\|v\|_{1} \leq\left\|\hat{\gamma}_{C}^{-1}\right\|\left\|\gamma_{C}(v)\right\|_{1 / 2, \Gamma_{C}}\right\}
$$

In order to show that $V$ is a non-empty set, let $w \in H^{1 / 2}\left(\Gamma_{C}\right)$ and $v \in H^{1}\left(\Omega, \Gamma_{D}\right)$ with $\hat{\gamma}_{C}^{-1}(w)=[v]$. If $\bar{z} \in \operatorname{ker} \gamma_{C}$ such that $\|v-\bar{z}\|_{1}=\inf _{z \in \operatorname{ker} \gamma_{C}}\|v-z\|_{1}$ and $v^{*}:=v-\bar{z}$, we obtain

$$
\gamma_{C}\left(v^{*}\right)=\gamma_{C}(v-\bar{z})=\gamma_{C}(v)=\hat{\gamma}_{C}([v])=w .
$$


Therefore, we have

$$
\left\|v^{*}\right\|_{1}=\inf _{z \in \operatorname{ker} \gamma_{C}}\|v-z\|_{1}=\left\|\hat{\gamma}_{C}^{-1}(w)\right\| \leq\left\|\hat{\gamma}_{C}^{-1}\right\|\|w\|_{1 / 2, \Gamma_{C}}=\left\|\hat{\gamma}_{C}^{-1}\right\|\left\|\gamma_{C}\left(v^{*}\right)\right\|_{1 / 2, \Gamma_{C}}
$$

which implies that $v^{*} \in V$. Moreover, there is a $v^{*} \in V$ for each $w \in H^{1 / 2}\left(\Gamma_{C}\right)$ such that (18) is valid, i.e., $\gamma_{C}(V)=H^{1 / 2}\left(\Gamma_{C}\right)$. Using these preparations, we conclude from the definition of the dual norm and Cauchy's inequality, that

$$
\begin{aligned}
\|\mu\|_{-1 / 2, \Gamma_{C}} & =\sup _{w \in H^{1 / 2}\left(\Gamma_{C}\right) \backslash\{0\}} \frac{\langle\mu, w\rangle}{\|w\|_{1 / 2, \Gamma_{C}}}=\sup _{v \in V \backslash\{0\}} \frac{\left\langle\mu, \gamma_{C}(v)\right\rangle}{\left\|\gamma_{C}(v)\right\|_{1 / 2, \Gamma_{C}}} \\
& =\sup _{v \in V \backslash\{0\}} \frac{\left(\nabla u^{\mu}, \nabla v\right)_{0}+\left(u^{\mu}, v\right)_{0}}{\left\|\gamma_{C}(v)\right\|_{1 / 2, \Gamma_{C}}} \leq \sup _{v \in V \backslash\{0\}} \frac{\left\|u^{\mu}\right\|_{1}\|v\|_{1}}{\left\|\gamma_{C}(v)\right\|_{1 / 2, \Gamma_{C}}} \\
& \leq\left\|\hat{\gamma}_{C}^{-1}\right\|^{-1}\left\|u^{\mu}\right\|_{1} .
\end{aligned}
$$

Setting $C_{1}:=\left\|\hat{\gamma}_{C}^{-1}\right\|$, we obtain the assertion.

Lemma 2 Let $\mu \in L^{2}\left(\Gamma_{C}\right)$ and $u^{\mu} \in H^{1}\left(\Omega, \Gamma_{D}\right) \cap H^{1+\theta}(\Omega)$ be the solution of (17), then there exists a function $u_{I}^{\mu} \in S^{p}\left(\mathscr{T}_{h}\right)$ and a constant $C_{2}>0$, independent of $u^{\mu}, h$ and $p$, such that

$$
\left\|u^{\mu}-u_{I}^{\mu}\right\|_{1} \leq C_{2} \frac{h^{\theta}}{p^{\theta}}\left\|u^{\mu}\right\|_{1+\theta}
$$

Proof. See [1, Thm. 4.6].

Lemma 3 There exists a constant $C_{3}>0$ which is independent of $H$ and $p_{C}$, such that

$$
\left\|\mu_{H}\right\|_{-1 / 2+\theta, \Gamma_{C}} \leq C_{3} \frac{\max \left\{1, p_{C}\right\}^{2 \theta}}{H^{\theta}}\left\|\mu_{H}\right\|_{-1 / 2, \Gamma_{C}}
$$

for all $\mu_{H} \in M^{p_{C}}$.

Proof. See [5, Thm. 3.5., Thm. 3.9] and (16).

We call the variational problem (17) regular, if $u^{\mu} \in H^{1}\left(\Omega, \Gamma_{D}\right) \cap H^{1+\theta}(\Omega)$ and

$$
\left\|u^{\mu}\right\|_{1+\theta} \leq C_{4}\|\mu\|_{-1 / 2+\theta, \Gamma_{C}}
$$

for all $\mu \in H^{-1 / 2+\theta}\left(\Gamma_{C}\right)$ and a constant $C_{4}>0$. Using Lemma 1, Lemma 2 and Lemma 3 as well as the regularity assumption (19) on $u^{\mu}$, we are able to prove the main theorem.

Theorem 4 Assume the variational problem (17) to be regular for $\theta \leq 1 / 2$ and

$$
\Pi\left(h, H, p, p_{C}\right):=\left(h H^{-1} \max \left\{1, p_{C}\right\}^{2} p^{-1}\right)^{\theta} \leq \varepsilon<C_{1}\left(C_{2} C_{3} C_{4}\right)^{-1}
$$

for some $\varepsilon>0$, then (11) holds for a constant $\alpha>0$ independent of $h, H, p$ und $p_{C}$. Proof. Let $\mu_{H} \in M^{p_{C}}\left(\mathscr{T}_{C, H}\right)$ and $u_{h}^{\mu_{H}} \in S^{p}\left(\mathscr{T}_{h}\right)$ be uniquely determined by

$$
\left(\nabla u_{h}^{\mu_{H}}, \nabla v_{h}\right)_{0}+\left(u_{h}^{\mu_{H}}, v_{h}\right)_{0}=\left(\mu_{H}, \gamma_{C}\left(v_{h}\right)\right)_{0, \Gamma_{C}}
$$


for all $v_{h} \in S^{p}\left(\mathscr{T}_{h}\right)$. Using the Galerkin orthogonality, Lemma 2, the regularity assumption and Lemma 3, we obtain

$$
\begin{aligned}
\left\|u^{\mu_{H}}-u_{h}^{\mu_{H}}\right\|_{1} & \leq\left\|u^{\mu_{H}}-u_{I}^{\mu_{H}}\right\|_{1} \leq C_{2} \frac{h^{\theta}}{p^{\theta}}\left\|u^{\mu_{H}}\right\|_{1+\theta} \leq C_{2} C_{4} \frac{h^{\theta}}{p^{\theta}}\left\|\mu_{H}\right\|_{-1 / 2+\theta, \Gamma_{C}} \\
& \leq C_{2} C_{3} C_{4}\left(\frac{h^{\theta}}{p^{\theta}} \frac{\max \left\{1, p_{C}\right\}^{2 \theta}}{H^{\theta}}\right)\left\|\mu_{H}\right\|_{-1 / 2, \Gamma_{C}} \\
& =C_{2} C_{3} C_{4} \Pi\left(h, H, p, p_{C}\right)\left\|\mu_{H}\right\|_{-1 / 2, \Gamma_{C}} .
\end{aligned}
$$

From Lemma 1, we obtain

$$
\begin{aligned}
\sup _{v_{h} \in S^{p}\left(\mathscr{T}_{h}\right) \backslash\{0\}} & \frac{\left(\mu_{H}, \gamma_{C}\left(v_{h}\right)\right)_{0, \Gamma_{C}}}{\left\|v_{h}\right\|_{1}} \geq \frac{\left(\mu_{H}, \gamma_{C}\left(u_{h}^{\mu_{H}}\right)\right)_{0, \Gamma_{C}}}{\left\|u_{h}^{\mu_{H}}\right\|_{1}}=\left\|u_{h}^{\mu_{H}}\right\|_{1} \\
& \geq\left\|u^{\mu_{H}}\right\|_{1}-\left\|u^{\mu_{H}}-u_{h}^{\mu_{H}}\right\|_{1} \geq C_{1}\left\|\mu_{H}\right\|_{-1 / 2, \Gamma_{C}}-\left\|u^{\mu_{H}}-u_{h}^{\mu_{H}}\right\|_{1} \\
& \geq\left(C_{1}-C_{2} C_{3} C_{4} \Pi\left(h, H, p, p_{C}\right)\right)\left\|\mu_{H}\right\|_{-1 / 2, \Gamma_{C}} \\
& \geq\left(C_{1}-C_{2} C_{3} C_{4} \varepsilon\right)\left\|\mu_{H}\right\|_{-1 / 2, \Gamma_{C}} .
\end{aligned}
$$

Setting $\alpha:=C_{1}-C_{2} C_{3} C_{4} \varepsilon>0$ yields the assertion.

From the pratical point of view, the result of Theorem 4 seems to be nonsatisfaying as it is not clear when $\Pi\left(h, H, p, p_{C}\right)$ is small enough such that (20) is fulfilled. Furthermore, it is often unclear whether the regularity assumption (19) holds. For convex domains, this assumption is fulfilled. Nevertheless, Theorem 4 justifies the modification of the discretization scheme by coarsening the mesh $\mathscr{T}_{C, H}$ or by decreasing the polynomial degree $p_{C}$ to obtain a stable scheme. In Section 7 , numerical results confirm this theoretical observation.

It remains to show that the discrete saddle point of (12) is also unique. Unfortunately, the inf-sup condition (11) does not fit to this problem. However, we can proceed in a similar way.

Lemma 4 Let $\tilde{L}^{2}\left(\Gamma_{C}\right):=\left\{\mu \in L^{2}\left(\Gamma_{C}\right) \mid \mu=0\right.$ on $\left.\Gamma_{C} \backslash \operatorname{supp} s\right\}$ and $C, C^{\prime}>0$. There exists a $\kappa>0$, such that for $h, H$, $p$ and $p_{C}$ satisfaying $\Pi\left(h, H, p, p_{C}\right)<\kappa$ there holds

$$
C\left\|s \mu_{H}\right\|_{-1 / 2, \Gamma_{C}}-C^{\prime} \Pi\left(h, H, p, p_{C}\right)\left\|\mu_{H}\right\|_{-1 / 2, \Gamma_{C}} \geq \kappa\left\|\mu_{H}\right\|_{-1 / 2, \Gamma_{C}}
$$

for all $\mu_{H} \in M^{p_{C}} \cap \tilde{L}^{2}\left(\Gamma_{C}\right)$.

Proof. Assume that for all $\kappa>0$ there exist $h_{\kappa}, H_{\kappa}, p_{\kappa}$ and $p_{C, \kappa}$ such that

$$
\Pi_{\kappa}:=\Pi\left(h_{\kappa}, H_{\kappa}, p_{\kappa}, p_{C, \kappa}\right)<\kappa
$$

and there exists a function $\mu_{\kappa} \in M^{p_{C, \kappa}}\left(\mathscr{T}_{C, H_{K}}\right) \cap \tilde{L}^{2}\left(\Gamma_{C}\right)$, such that

$$
C\left\|s \mu_{\kappa}\right\|_{-1 / 2, \Gamma_{C}}-C^{\prime} \Pi_{\kappa}\left\|\mu_{\kappa}\right\|_{-1 / 2, \Gamma_{C}}<\kappa\left\|\mu_{\kappa}\right\|_{-1 / 2, \Gamma_{C}} .
$$

Obviously, $\mu_{\kappa} \neq 0$. Defining $\tilde{\mu}_{\kappa}:=\left\|\mu_{\kappa}\right\|_{0, \Gamma_{C}}^{-1} \mu_{\kappa} \in \tilde{L}^{2}\left(\Gamma_{C}\right)$, we obtain $\left\|\tilde{\mu}_{\kappa}\right\|_{0, \Gamma_{C}}=1$. Due to the reflexivity of $L^{2}\left(\Gamma_{C}\right)$ and the convexity as well as the closeness of $\tilde{L}^{2}\left(\Gamma_{C}\right)$, there exists some $\tilde{\mu} \in \tilde{L}^{2}\left(\Gamma_{C}\right)$ such that $\tilde{\mu}_{\kappa_{n}} \rightarrow \tilde{\mu}$ for a sequence $\kappa_{n} \rightarrow 0$. This also implies $\tilde{\mu}_{\kappa_{n}} \rightarrow \tilde{\mu}$ in the norms $\|\cdot\|_{0, \Gamma_{C}}$ and $\|\cdot\|_{-1 / 2, \Gamma_{C}}$. Therefore, $\|\tilde{\mu}\|_{0, \Gamma_{C}}=1$ and $\tilde{\mu} \neq 0$ on $\operatorname{supp} s$. From (21), we have $C\left\|s \tilde{\mu}_{\kappa_{n}}\right\|_{-1 / 2, \Gamma_{C}}<\left(1+C^{\prime}\right) \kappa_{n}$ which implies $\|s \tilde{\mu}\|_{-1 / 2, \Gamma_{C}}=0$ and therefore, $s \tilde{\mu}=0$, which is a contradiction to $\tilde{\mu} \neq 0$ on supp $s . \square$ 
Theorem 5 Let the variational problem (17) be regular for $\theta \leq 1 / 2$ and $s \in L^{\infty}\left(\Gamma_{C}\right)$. Furthermore, let $\Pi\left(h, H, p, p_{C}\right)$ be sufficiently small. Then, there exists a constant $\alpha>0$ sucht that

$$
\alpha\left\|\mu_{H}\right\|_{-1 / 2, \Gamma_{C}} \leq \sup _{\substack{v_{h} \in S^{p}\left(\mathscr{T}_{h}\right) \\\left\|v_{h}\right\|_{1}=1}}\left(\mu_{H}, s \gamma_{C}\left(v_{h}\right)\right)_{0, \Gamma_{C}}
$$

for all $\mu_{H} \in M^{p_{C}}\left(\mathscr{T}_{C, H}\right) \cap \tilde{L}^{2}\left(\Gamma_{C}\right)$, where $\alpha$ is independent of $h, H$, $p$ und $p_{C}$.

Proof. Let $\mu_{H} \in M^{p_{C}}\left(\mathscr{T}_{C, H}\right) \cap \tilde{L}^{2}\left(\Gamma_{C}\right)$. Furthermore, let $u_{h}^{s \mu_{H}} \in S^{p}\left(\mathscr{T}_{h}\right)$ be uniquely determined by

$$
\forall v_{h} \in S^{p}\left(\mathscr{T}_{h}\right):\left(\nabla u_{h}^{\mu_{H}}, \nabla v_{h}\right)_{0}+\left(u_{h}^{\mu_{H}}, v_{h}\right)_{0}=\left(s \mu_{H}, \gamma_{C}\left(v_{h}\right)\right)_{0, \Gamma_{C}} .
$$

Thus, in analogy to the proof of Theorem 4, we obtain

$$
\begin{aligned}
\left\|u^{s \mu_{H}}-u_{h}^{s \mu_{H}}\right\|_{1} & \leq C_{2} C_{4} \frac{h^{\theta}}{p^{\theta}}\left\|s \mu_{H}\right\|_{-1 / 2+\theta, \Gamma_{C}} \leq C_{2} C_{4} \frac{h^{\theta}}{p^{\theta}}\|s\|_{\infty, \Gamma_{C}}\left\|\mu_{H}\right\|_{-1 / 2+\theta, \Gamma_{C}} \\
& \leq C_{2} C_{3} C_{4}\|s\|_{\infty, \Gamma_{C}} \Pi\left(h, H, p, p_{C}\right)\left\|\mu_{H}\right\|_{-1 / 2}
\end{aligned}
$$

and

$$
\begin{aligned}
\sup _{v_{h} \in S^{p}\left(\mathscr{T}_{h}\right) \backslash\{0\}} \frac{\left(s \mu_{H}, \gamma_{C}\left(v_{h}\right)\right)_{0, \Gamma_{C}}}{\left\|v_{h}\right\|_{1}} & \\
& \geq C_{1}\left\|s \mu_{H}\right\|_{-1 / 2, \Gamma_{C}}-C_{2} C_{3} C_{4}\|s\|_{\infty, \Gamma_{C}} \Pi\left(h, H, p, p_{C}\right)\left\|\mu_{H}\right\|_{-1 / 2} .
\end{aligned}
$$

The appliance of Lemma 4 completes the proof.

Corollary 1 The discrete saddle point of (12) is unique, if $\Pi\left(h, H, p, p_{C}\right)$ is sufficiently small.

Proof. The assertion follows analogously to the proof of Theorem 3, using (22).

\section{Numerical results}

In our numerical experiments, we study the simplified Signorini problem and the idealized frictional problem with $\Omega:=(-1,1)^{2}, \Gamma_{C}:=(-1,1) \times\{-1\}, \Gamma_{D}:=[-1,-1] \times$ $\{1\} \cup\{1\} \times[0,1]$ and $f:=-1$. For the simplified Signorini problem, we define the obstacle function as $g\left(x_{0}, x_{1}\right):=-x_{0}^{2}$. In Figure 1, the finite element solution $u$ of the simplified Signorini problem is depicted. In addition, the obstacle function $g$ and the Lagrange multiplier $\lambda_{0}$ are sketched in. We observe, that the condition $u \geq g$ is fulfilled. For $u \in H^{1}\left(\Omega, \Gamma_{D}\right) \cap H^{2}(\Omega)$, there holds $\lambda_{0} \in L^{2}\left(\Gamma_{C}\right)$ and $\lambda_{0}=-\partial_{n} u$. Thus, we have $\lambda_{0}(u-g)=0$ on $\Gamma_{C}$. This condition can also be seen in Figure 1. In Figure $2, \lambda_{0, H}$ is depicted for $p \equiv 1, p_{C} \equiv 0$ and different quotients of the mesh sizes $h$ and $H$. In Figure 2(a) the quotient is chosen as $h / H=1$. The Lagrange multiplier seems to oscillate. This oscillation phenomena can be interpreted as an one-dimensional checkerboard instability, which suggests that the Lagrange multiplier is not unique. In this case $\lambda_{0, H}$ is not a reasonable approximation of $-\partial_{n} u$. As suggested by Theorem 4 , the uniqueness of the Lagrange multiplier is obtained by reducing $\Pi\left(h, H, p, p_{C}\right)$. 


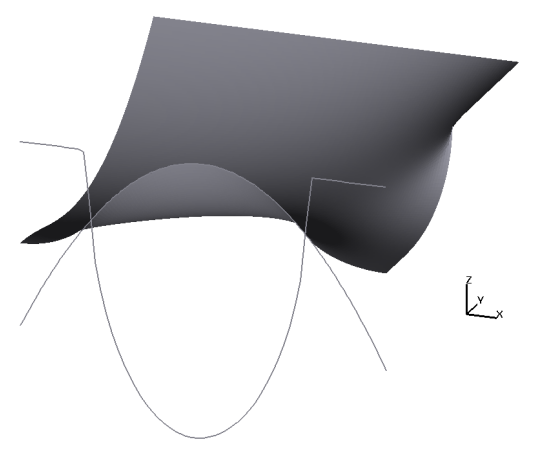

Fig. 1 Solution $u$ of the simplified Signorini problem with obstacle function $g$ and Lagrange multiplier $\lambda_{0}$ on the boundary.

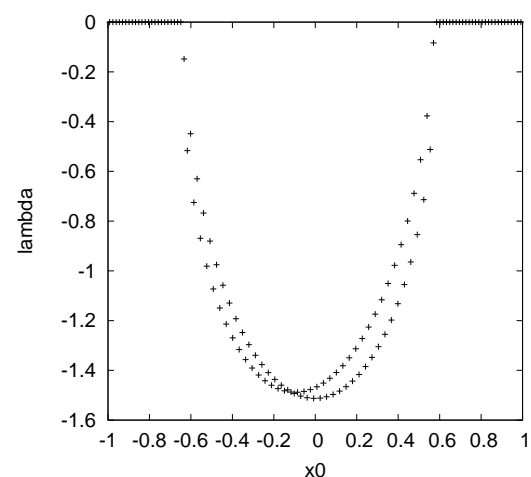

(a)

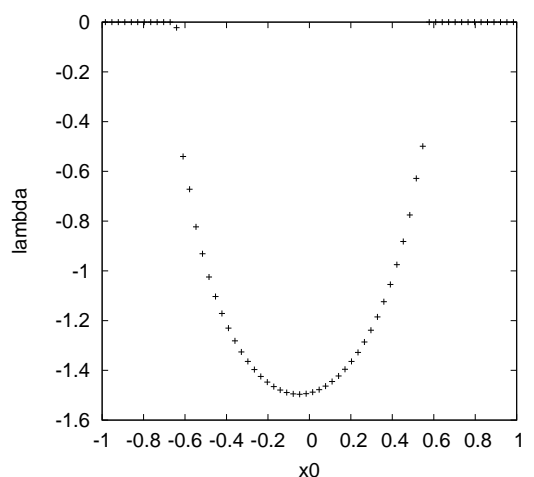

(b)

Fig. $2 \lambda_{0, H}$ for (a) $p \equiv 1, p_{C} \equiv 0, h / H=1$, (b) $p \equiv 1, p_{C} \equiv 0, h / H=0.5$.

Indeed, for $h / H=0.5$ the described patterns of instability do not occur, see Figure 2(b). It is noted that the use of meshes $\mathscr{T}_{h}$ and $\mathscr{T}_{C, h}$ with different mesh sizes $h$ and $H$ lead to high complexity in implementation. For the edge grid $\mathscr{T}_{C, H}$ which is inherited from $\mathscr{T}_{h}$, the implementational effort is essentially smaller. However, this enforces sizes $h$ and $H$ with $h / H=1$. In order to keep $\Pi\left(h, H, p, p_{C}\right)$ small in this case, we can vary $p$ and $p_{C}$. In Figure $3(\mathrm{a}), \lambda_{0, H}$ is depicted for $p \equiv 2, p_{C} \equiv 1$. Obviously, there are no instability patterns for this combination, whereas the combination $p \equiv 3$, $p_{C} \equiv 2$ and $h / H=1$ leads to a Lagrange multiplier with instability patterns, cf. Figure 3(b). The use of $h / H=0.5$ or $p_{C} \equiv 1$ avoids these patterns, see Figures 3(c) and (d). Further experiments show that the combination $h / H=1, p_{C} \equiv p-1$ for even polynomial degree $p$ leads to Lagrange multipliers without instability patterns. For odd polynomial degree $p$ we have to choose $h / H=0.5$ in order to avoid such patterns. It is noted that the presence or absence of instability patterns do not strictly verify or falsify the unique existence of the Lagrange multliplier. However, such patterns can be seen as an indication for the non-uniqueness of the Lagrange multiplier. The 


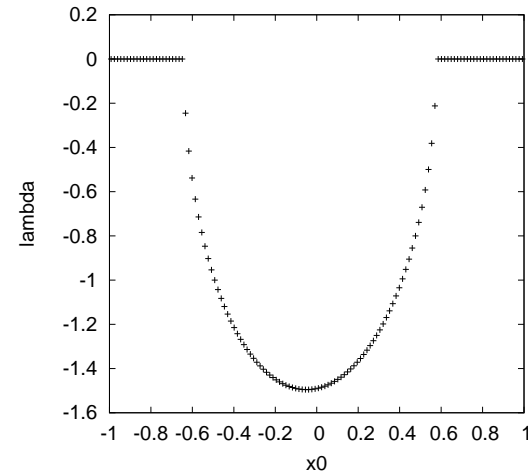

(a)

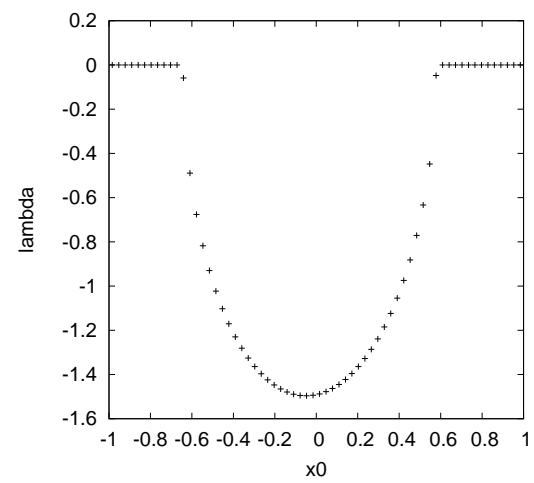

(c)

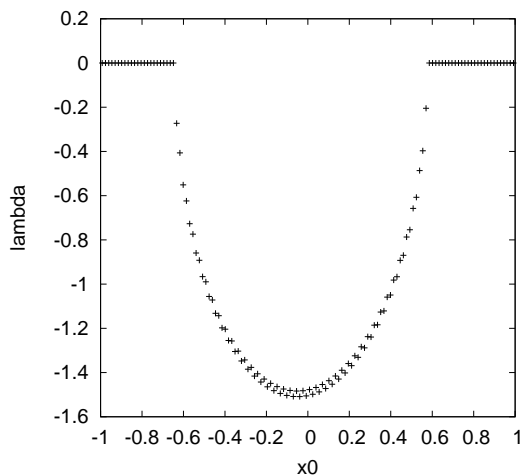

(b)

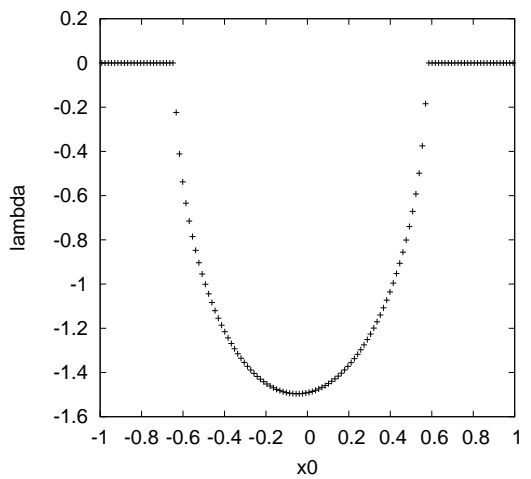

(d)

Fig. $3 \lambda_{0, H}$ for (a) $p \equiv 2, p_{C} \equiv 1, h / H=1$, (b) $p \equiv 3, p_{C} \equiv 2, h / H=1$, (c) $p \equiv 3, p_{C} \equiv 2, h / H=0.5$, (d) $p \equiv 3, p_{C} \equiv 1, h / H=1$.

effects resulting from varying $\Pi\left(h, H, p, p_{C}\right)$ confirm this observation. For the idealized frictional problem, we set $s:=\left(1-x_{0}\right)^{2}$. The solution $u$ is depicted in Figure 4. The constant function 1 and the Lagrange multiplier $\lambda_{1}$ are also sketched in. For $u \in H^{1}\left(\Omega, \Gamma_{D}\right) \cap H^{2}(\Omega)$, we obtain $s \lambda_{1}=-\partial_{n} u$ which implies $\left|\lambda_{1}\right| \leq 1$. Furthermore, $\left|\lambda_{1}\right|<1$ yields $u=0$. For $\left|\lambda_{1}\right|=1$, we find that $u \leq 0$. These relations can also be seen in Figure 4.

In Figure 5, the Lagrange multiplier $\lambda_{1, H}$ is depicted for various qoutients $h / H$ and polynomial degrees $p$ und $p_{C}$. We obtain similar results as for the simplified Signorini problem. In Figure 6, the solution $u$ of the alternative formulation of the idealized frictional problem is depicted. Moreover, the function $s$ and the Lagrange multiplier $\lambda_{1}$ are sketched in. We obtain $\lambda_{1}=-\partial_{n} u$ for $u \in H^{1}\left(\Omega, \Gamma_{D}\right) \cap H^{2}(\Omega)$ and, therefore, $\left|\lambda_{1}\right| \leq s$. Here, $\left|\lambda_{1}\right|<s$ implies $u=0$ and $\left|\lambda_{1}\right|=s$ implies $u \leq 0$. These relations can be seen in Figure 6.

Since the discrete inf-sup condition (14) has to be considered for this problem, we obtain the same results as for the simplified Signorini problem. In Figure 7, the La- 

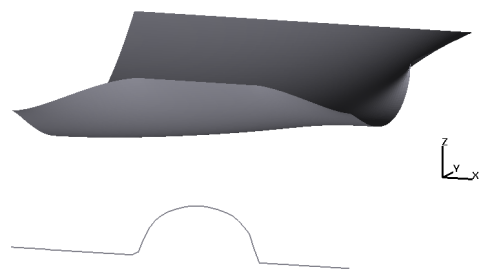

Fig. 4 Solution $u$ of the idealized frictional problem with function 1 and Lagrange multiplier $\lambda_{1}$ on the boundary.

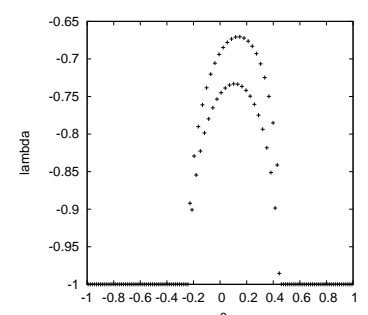

(a)

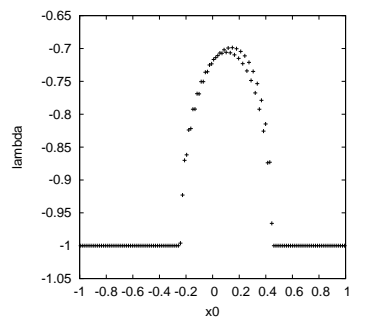

(d)

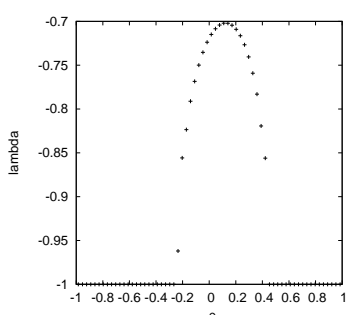

(b)

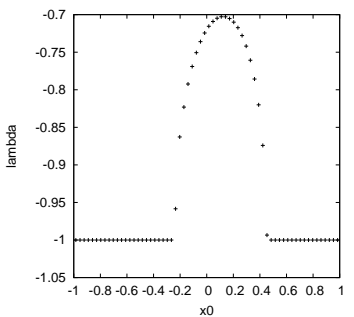

(e)

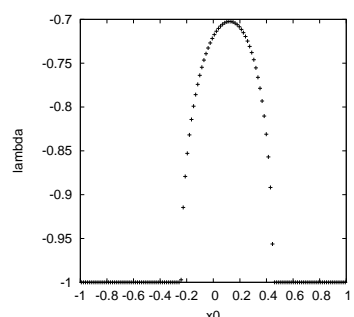

(c)

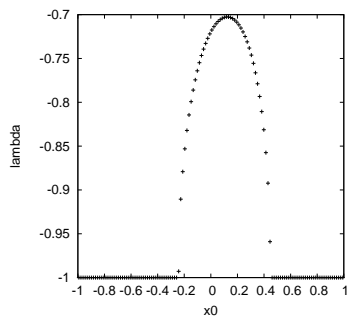

(f)

Fig. $5 \lambda_{1, H}$ for (a) $p \equiv 1, p_{C} \equiv 0, h / H=1$, (b) $p \equiv 1, p_{C} \equiv 0, h / H=0.5$, (c) $p \equiv 2, p_{C} \equiv 1, h / H=1$, (d) $p \equiv 3, p_{C} \equiv 2, h / H=1$, (e) $p \equiv 3, p_{C} \equiv 2, h / H=0.5$, (f) $p \equiv 3, p_{C} \equiv 1, h / H=1$.

grange multiplier $\lambda_{1, H}$ is depicted for various quotients $h / H$ and polynomial degrees $p$ und $p_{C}$.

Remark 3 From the practical point of view, it is crucial to ensure higher-order finite element functions to be in $M_{-}^{p_{C}}\left(\mathscr{T}_{C, H}\right)$ and $M_{1}^{p_{C}}\left(\mathscr{T}_{C, H}\right)$ for $p_{C, T_{C}} \geq 2$. For $M_{S}^{p_{C}}\left(\mathscr{T}_{C, H}\right)$ 


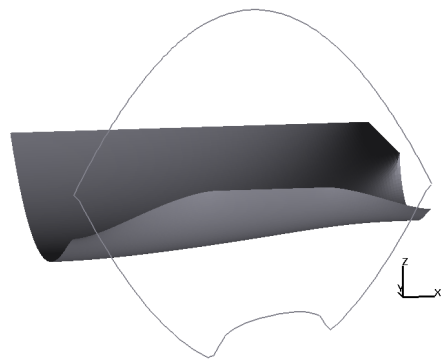

Fig. 6 Solution $u$ of the alternative formulation of the idealized frictional problem with function $s$ and Lagrange multiplier $\lambda_{1}$ on the boundary.

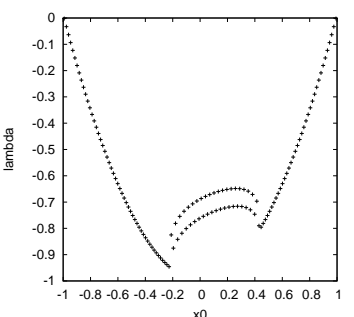

(a)

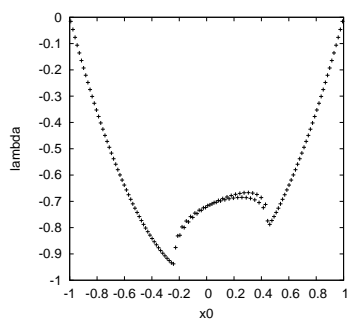

(d)

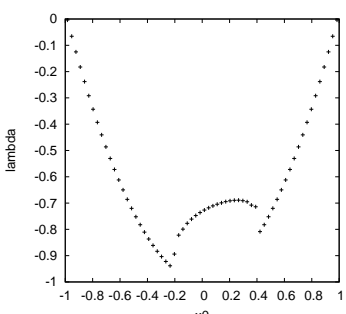

(b)

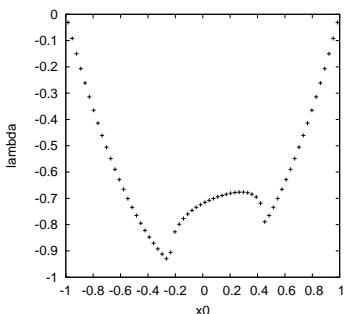

(e)

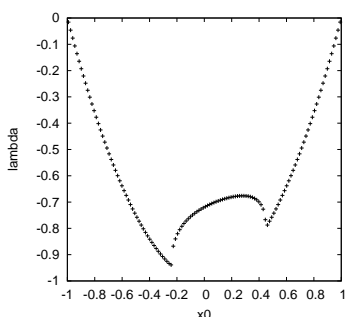

(c)

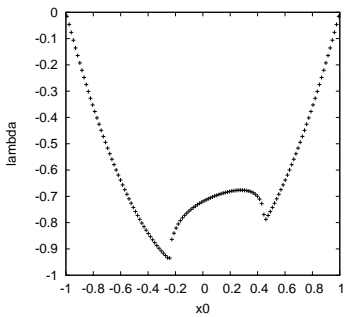

(f)

Fig. $7 \quad \lambda_{1, H}$ for (a) $p \equiv 1, p_{C} \equiv 0, h / H=1$, (b) $p \equiv 1, p_{C} \equiv 0, h / H=0.5$, (c) $p \equiv 2, p_{C} \equiv 1, h / H=1$, (d) $p \equiv 3, p_{C} \equiv 2, h / H=1$, (e) $p \equiv 3, p_{C} \equiv 2, h / H=0.5$, (f) $p \equiv 3, p_{C} \equiv 1, h / H=1$.

it is already cruical for $p_{C, T_{C}} \geq 0$. It is reasonable to replace these sets by

$$
\begin{array}{r}
\tilde{M}_{-}^{p_{C}}\left(\mathscr{T}_{C, H}\right):=\left\{\mu_{H} \in M^{p_{C}}\left(\mathscr{T}_{C, H}\right) \mid \forall T \in \mathscr{T}_{C, H}: \forall x \in \mathscr{C}: \mu_{H \mid T}\left(\Psi_{C, T}(x)\right) \leq 0\right\}, \\
\tilde{M}_{1}^{p_{C}}\left(\mathscr{T}_{C, H}\right):=\left\{\mu_{H} \in M^{p_{C}}\left(\mathscr{T}_{C, H}\right)\left|\forall T \in \mathscr{T}_{C, H}: \forall x \in \mathscr{C}:\right| \mu_{H \mid T}\left(\Psi_{C, T}(x)\right) \mid \leq 1\right. \\
\text { on supp } \left.s, \mu_{H \mid T}\left(\Psi_{C, T}(x)\right)=0 \text { on } \Gamma_{C} \backslash \operatorname{supp} s\right\}, \\
\tilde{M}_{s}^{p_{C}}\left(\mathscr{T}_{C, H}\right):=\left\{\mu_{H} \in M^{p_{C}}\left(\mathscr{T}_{C, H}\right)\left|\forall T \in \mathscr{T}_{C, H}: \forall x \in \mathscr{C}:\right| \mu_{H \mid T}\left(\Psi_{C, T}(x)\right) \mid \leq s(x)\right\}
\end{array}
$$


where $\mathscr{C} \subset[-1,1]^{k-1}$ is a sufficiently large set of discrete points. We use Chebycheff points to ensure the additional error to be small. We refer to [3] for a further justification of this approach.

\section{References}

1. Babuska, I., Suri, M.: The h-p version of the finite element method with quasiuniform meshes. $\mathbf{M}^{2} \mathrm{AN}$ 21, 199-238 (1987)

2. Brezzi, F., Hager, W.W., Raviart, P.A.: Error estimates for the finite element solution of variational inequalities. part i. primal theory. Numer. Math. 28, 431-443 (1977)

3. Ehlich, H., Zeller, K.: Schwankungen von polynomen zwischen gitterpunkten. Math. Z. 86, 41-44 (1964)

4. Ekeland, I., Temam, R.: Convex analysis and variational problems. Studies in Mathematics and its Applications. North-Holland Publishing Company, Amsterdam (1976)

5. Georgoulis, E.H.: Inverse-type estimates on $h p$-finite element spaces and applications. Math. Comput 77(261), 201-219 (2008). DOI 10.1090/S0025-5718-07-02068-6

6. Glowinski, R.: Numerical methods for nonlinear variational problems. Springer Series in Computational Physics. Springer-Verlag, New York (1984)

7. Glowinski, R., Lions, J.L., Trémolierès, R.: Numerical analysis of variational inequalities. Studies in Mathematics and its Applications. North-Holland Publishing Company, Amsterdam (1981)

8. Haslinger, J.: Mixed formulation of elliptic variational inequalities and its approximation. Apl. Mat 26, 462-475 (1981)

9. Haslinger, J., Lovisek, J.: Mixed variational formulation of unilateral problems. Commentat. Math. Univ. Carol. 21, 231-246 (1980)

10. Hlávaček, I., Haslinger, J., Nečas, J., Lovíšek, J.: Solution of variational inequalities in mechanics. Applied Mathematical Sciences. Springer-Verlag, New York (1988)

11. Kikuchi, N., Oden, J.: Contact problems in elasticity: A study of variational inequalities and finite element methods. SIAM Studies in Applied Mathematics. SIAM, Society for Industrial and Applied Mathematics, Philadelphia (1988)

12. Krebs, A.: On solving nonlinear variational inequalities by $p$-version finite elements. Ph.D. thesis, Fachbereich Mathematik der Universität Hannover (2004)

13. Krebs, A., Stephan, E.P.: A p-version finite element method for nonlinear elliptic variational inequalities in 2D. Numer. Math. 105(3), 457-480 (2007). DOI 10.1007/s00211-006-0035-0

14. Lions, J.L., Magenes, E.: Non-homogeneous boundary value problems and applications. Vol. I. Translated from the French by P. Kenneth. Die Grundlehren der mathematischen Wissenschaften. SpringerVerlag (1972)

15. Oden, J., Kikuchi, N.: Finite element methods for constrained problems in elasticity. Int. J. Numer. Methods Eng. 18, 701-725 (1982). DOI 10.1002/nme.1620180507

16. Triebel, H.: Interpolation theory, function spaces, differential operators. North-Holland Mathematical Library. North-Holland Publishing Company, Amsterdam (1978)

17. Yosida, K.: Functional analysis. Grundlehren der mathematischen Wissenschaften. Springer-Verlag, Berlin (1980) 